\title{
Tunable phase control for subluminal to superluminal light propagation
}

\author{
Mostafa Sahrai, ${ }^{1}$ Habib Tajalli, ${ }^{1}$ Kishore T. Kapale, ${ }^{2}$ and M. Suhail Zubairy ${ }^{2,3}$ \\ ${ }^{1}$ Department of Physics, Tabriz University, Tabriz, Iran \\ ${ }^{2}$ Institute for Quantum Studies and Department of Physics, Texas A \& M University, College Station, Texas 77843-4242, USA \\ ${ }^{3}$ Department of Electronics, Quaid-i-Azam University, Islamabad, Pakistan \\ (Received 9 April 2004; published 24 August 2004)
}

\begin{abstract}
We demonstrate tunable control of the group velocity of a weak probe pulse from subluminal to superluminal. The model is an extended $\Lambda$-type system with two extra control fields and an extra energy level. Phase variation of one of the control fields imparts the tunability in the group velocity along with other interesting spectral behavior in the absorption spectrum.
\end{abstract}

DOI: 10.1103/PhysRevA.70.023813

PACS number(s): 42.50.Gy, 42.62.Fi

In recent years, a remarkable control of the group velocity of light in material systems has been demonstrated experimentally leading to both ultraslow and superluminal propagation of light [1]. The early studies in superluminal light propagation involved pulse propagation through an amplifying medium. The optical saturation effect is responsible for pulse advancement as the front edge of the pulse depletes the atomic inversion and the trailing edge sees a medium with small gain [2]. More recently, gain assisted anomalous dispersion leading to superluminal light propagation is demonstrated by making use of a pair of Raman gain features via a bi-chromatic driving beam in electromagnetically induced transparency (EIT) [3,4]. In another experiment, superluminal propagation is observed by beating the pump and the probe field to generate coherent population pulsations in the medium [5]. The suggestion for ultraslow light involving large dispersion at zero absorption in EIT [6] was implemented using ultracold gases [7]. Shortly thereafter, two groups reported ultraslow light in hot gases [8,9] followed by a demonstration in solids $[5,10]$.

In view of many potential applications of ultraslow and superluminal light propagation, a question of interest is whether one can have a controlling parameter in a single experiment for switching from subluminal to superluminal propagation. In this paper we propose a scheme based on four-level EIT in which we can switch the propagation of light from subluminal to superluminal by changing the phase of one of the driving fields. In contrast to some earlier schemes with a controlling parameter for the switching from subluminal to superluminal light propagation [11-13] in closed three-level $\Lambda$ systems, we consider an open system. To note, in an interesting article by Wicht et al. [14] a variety of open and closed systems have been studied and compared for achieving superluminality with minimal absorption.

The motivation to consider such a system comes from our recent studies on the phase sensitivity of spontaneous emission spectrum in such a four-level system [15] leading to a variety of interesting effects such as line narrowing and partial cancellation of spontaneous emission. In another study, such phase sensitivity has been used in subwavelength atom localization in a region spanned by half the wavelength [16].

To illustrate the advantages of our model we note that in the proposal by the Friedmann group [11] superluminality is accompanied by considerable absorption. Whereas in our scheme within some parameter range the absorption is considerably reduced increasing the practical applicability of the scheme. The proposal by Agarwal et al. [13] is notable for intensity control over the group velocity where the superluminality is accompanied by gain as in the experimental observation by Wang et al. [4] and the theoretical proposal by Wilson-Gordon et al. [12]. We observe that the intensity control of group velocity exists in our system as well along with its switching from subluminal to superluminal by simple variation of the phase of one of the fields. To point out another advantage of our model we note that Goren et al. [17] have found that the switchover in the sign of the dispersion is only observable in the absence of Doppler broadening in the models they considered. Our model is naturally Doppler free when all the fields are co-propagating. We discuss the relevant details toward the end of the paper.

In the proposed scheme (see Fig. 1) the upper level of the probe field transition is coupled to two levels in cascade configuration as in systems demonstrating double dark resonances [18]. However, In addition to the two driving fields coupling the $\left|a_{1}\right\rangle \rightarrow|b\rangle$ and $\left|a_{2}\right\rangle \rightarrow|b\rangle$ transitions we have another driving field that couples the dipole forbidden $\left|a_{1}\right\rangle$ $\rightarrow\left|a_{2}\right\rangle$ transition. It is the phase of this driving field that affects the medium dispersion in such a way that we can have a switch from subluminal to superluminal group velocity.

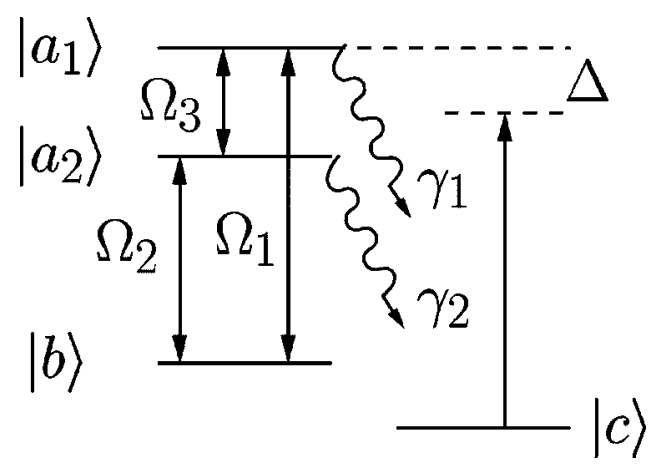

FIG. 1. The energy-level scheme. We manipulate the coherence through three fields. $\Omega_{1}$ and $\Omega_{3}$ have a definite phase relationship that we control to manipulate the probe absorption of the fourth field $\Delta$ detuned from the $\left|a_{1}\right\rangle-|c\rangle$ transition. 
The Hamiltonian for the problem can be written as

$$
\mathcal{H}=\mathcal{H}_{0}+\mathcal{H}_{I}
$$

where the self-energy $\mathcal{H}_{0}$ is given by

$$
\mathcal{H}_{0}=\hbar \omega_{a_{1}}\left|a_{1}\right\rangle\left\langle a_{1}\left|+\hbar \omega_{a_{2}}\right| a_{2}\right\rangle\left\langle a_{2}\left|+\hbar \omega_{b}\right| b\right\rangle\left\langle b\left|+\hbar \omega_{c}\right| c\right\rangle\langle c|,
$$

and the interaction Hamiltonian is

$$
\begin{aligned}
\mathcal{H}_{I}= & -\frac{\hbar}{2}\left[\Omega_{1} e^{-i \nu_{1} t}\left|a_{1}\right\rangle\left\langle b\left|+\Omega_{2} e^{-i \nu_{2} t}\right| a_{2}\right\rangle\left\langle b\left|+\Omega_{3} \mathrm{e}^{-i \nu_{3} t}\right| a_{1}\right\rangle\left\langle a_{2}\right|\right. \\
& \left.+\Omega_{p} e^{-i \nu_{p} t}\left|a_{1}\right\rangle\langle c|+\text { H.c. }\right] .
\end{aligned}
$$

Here, $\omega_{i}$ correspond to the energy of state $|i\rangle$; the angular frequencies of the optical fields are denoted by $\nu_{i}$; and the subscript $p$ stands for the quantity corresponding to the probe field. For the rest of the discussion we assume the Rabi frequencies $\Omega_{1}, \Omega_{2}$ to be real and allow $\Omega_{3}$ to have a carrying phase, i.e., $\Omega_{3}=\left|\Omega_{3}\right| e^{-i \phi}$. Since the three driving fields form a closed loop the phase can be imparted to any one of them and that will not change the result of the calculation. This will become clear at a later stage. The coupling of the probe field of amplitude $\mathcal{E}_{p}$ is governed by the corresponding Rabi frequency $\Omega_{p}=\mathcal{E}_{p} \wp_{a_{1} c} / \hbar$. We note that $\wp_{a_{1} c}$ is the dipole moment associated with the transition $\left|a_{1}\right\rangle-|c\rangle$.

We construct the density-matrix equations through

$$
\dot{\rho}=-\frac{i}{\hbar}[H, \rho]-\frac{1}{2}\{\Gamma, \rho\}
$$

where $\{\Gamma, \rho\}=\Gamma \rho+\rho \Gamma$. Here the decay rates are incorporated into the equations through the decay matrix $\Gamma$, which are defined by $\langle n|\Gamma| m\rangle=\gamma_{n} \delta_{n m}$. The detailed form of these equations are given in the Appendix.

After straightforward algebraic manipulation and transformation to appropriate rotating frames we obtain a set of density-matrix equations. The most important equations from this set are

$$
\begin{gathered}
\dot{\tilde{\rho}}_{a_{1} c}=-\left(i \Delta+\frac{\gamma_{1}}{2}\right) \tilde{\rho}_{a_{1} c}+\frac{i}{2} \Omega_{3} \tilde{\rho}_{a_{2} c}+\frac{i}{2} \Omega_{1} \tilde{\rho}_{b c} \\
-\frac{i}{2} \Omega_{p}\left(\tilde{\rho}_{a_{1} a_{1}}-\tilde{\rho}_{c c}\right), \\
\dot{\tilde{\rho}}_{a_{2} c}=-\left(i \Delta+\frac{\gamma_{2}}{2}\right) \tilde{\rho}_{a_{2} c}+\frac{i}{2} \Omega_{3}^{*} \widetilde{\rho}_{a_{1} c}+\frac{i}{2} \Omega_{2} \widetilde{\rho}_{b c}-\frac{i}{2} \Omega_{p} \tilde{\rho}_{a_{2} a_{1}}, \\
\dot{\tilde{\rho}}_{b c}=-\left(i \Delta+\gamma_{b c}\right) \tilde{\rho}_{b c}+\frac{i}{2} \Omega_{1} \widetilde{\rho}_{a_{1} c}+\frac{i}{2} \Omega_{2} \widetilde{\rho}_{a_{2} c}-\frac{i}{2} \Omega_{p} \widetilde{\rho}_{b a_{1}} .
\end{gathered}
$$

At this point we use the fact that the probe field is weak compared to the other three driving fields. We keep terms of all orders in the strong driving fields, but only the terms which are linear in the probe field. This amounts to using

$$
\tilde{\boldsymbol{\rho}}_{c c}^{(0)}=1, \quad \tilde{\rho}_{b a_{1}}^{(0)}=0, \quad \tilde{\rho}_{a_{1} a_{1}}^{(0)}=0, \quad \tilde{\rho}_{a_{2} a_{1}}^{(0)}=0 .
$$

Thus we obtain

$$
\begin{gathered}
\dot{\tilde{\rho}}_{a_{1} c}=-\left(i \Delta+\frac{\gamma_{1}}{2}\right) \tilde{\rho}_{a_{1} c}+\frac{i}{2} \Omega_{3} \widetilde{\rho}_{a_{2} c}+\frac{i}{2} \Omega_{1} \tilde{\rho}_{b c}+\frac{i}{2} \Omega_{p}, \\
\dot{\tilde{\rho}}_{a_{2} c}=-\left(i \Delta+\frac{\gamma_{2}}{2}\right) \widetilde{\rho}_{a_{2} c}+\frac{i}{2} \Omega_{3}^{*} \widetilde{\rho}_{a_{1} c}+\frac{i}{2} \Omega_{2} \widetilde{\rho}_{b c}, \\
\dot{\tilde{\rho}}_{b c}=-i \Delta \tilde{\rho}_{b c}+\frac{i}{2} \Omega_{1} \tilde{\rho}_{a_{1} c}+\frac{i}{2} \Omega_{2} \tilde{\rho}_{a_{2} c},
\end{gathered}
$$

where we have also substituted $\gamma_{b c}=0$. Notice that this particular subset of equations forms a complete set and can thus be treated independently of the other density-matrix equations. This simplifies the process of obtaining analytic solutions.

The above equations can be solved in a straightforward manner following the recipe discussed in the Appendix to obtain

$$
\begin{aligned}
\rho_{a_{1} c}= & \tilde{\rho}_{a_{1} c} \exp \left(-i \nu_{p} t\right)=\frac{1}{Y \hbar}\left(\Omega_{2}^{2}-4 \Delta^{2}+i 2 \gamma_{2} \Delta\right) \mathcal{E}_{p} \wp_{a_{1} c} \\
& \times \exp \left(-i \nu_{p} t\right),
\end{aligned}
$$

where $Y=A+i B$, with

$$
\begin{aligned}
A= & -8 \Delta^{3}+2 \Delta\left(\Omega_{1}^{2}+\Omega_{2}^{2}+\Omega_{3}^{2}\right)+2 \gamma_{1} \gamma_{2} \Delta \\
& +\Omega_{1} \Omega_{2} \Omega_{3}\left(e^{i \phi}+e^{-i \phi}\right), \\
& B=4 \Delta^{2}\left(\gamma_{1}+\gamma_{2}\right)-\left(\gamma_{1} \Omega_{2}^{2}+\gamma_{2} \Omega_{1}^{2}\right) .
\end{aligned}
$$

Note now that the susceptibility can be written as

$$
\chi=\frac{2 N \wp_{c a_{1}} \rho_{a_{1} c}}{\epsilon_{0} \mathcal{E}_{p}} e^{i \nu_{p} t}=\frac{2 N\left|\wp_{a_{1}}\right|^{2}}{\epsilon_{0}} \frac{\left(\Omega_{2}^{2}-4 \Delta^{2}+i 2 \gamma_{2} \Delta\right)}{Y \hbar},
$$

where $N$ is the atom number density in the medium. Separating the real and imaginary parts $\chi=\chi^{\prime}+i \chi^{\prime \prime}$, we obtain

$$
\begin{aligned}
& \chi^{\prime}=\frac{2 N\left|\wp_{a_{1} c}\right|^{2}}{\epsilon_{0} \hbar Z}\left\{\left(\Omega_{2}^{2}-4 \Delta^{2}\right) A+2 \gamma_{2} \Delta B\right\}, \\
& \chi^{\prime \prime}=\frac{2 N\left|\wp_{a_{1} c}\right|^{2}}{\epsilon_{0} \hbar Z}\left\{2 \gamma_{2} \Delta A-\left(\Omega_{2}^{2}-4 \Delta^{2}\right) B\right\},
\end{aligned}
$$

where $Z=Y Y^{*}$. It is imperative to point out that the phase enters the susceptibility expression only through quantities $A$ and $Y$. Even the phase dependence of $Y$ is only through that of $A$. Also we observe that the phase dependent term in $A$ is $\Omega_{1} \Omega_{2} \Omega_{3}\left(e^{i \phi}+e^{-i \phi}\right)$. Thus this phase factor could very well have come from either of the three driving fields. Moreover, if all the fields had phase dependence, only the collective phase would be important and no individual phase-dependent terms would occur. This collective phase can be easily determined to be $\phi=\phi_{2}+\phi_{3}-\phi_{1}$ by repeating the susceptibility calculation and noting that the Rabi frequencies are complex in general. Here $\phi_{i}$ is the phase of the complex Rabi frequency $\Omega_{i}$ of the driving field.

For further discussion the relevant quantity to consider is the group index $n_{g}=c / v_{g}$, where $c$ is the speed of light in 

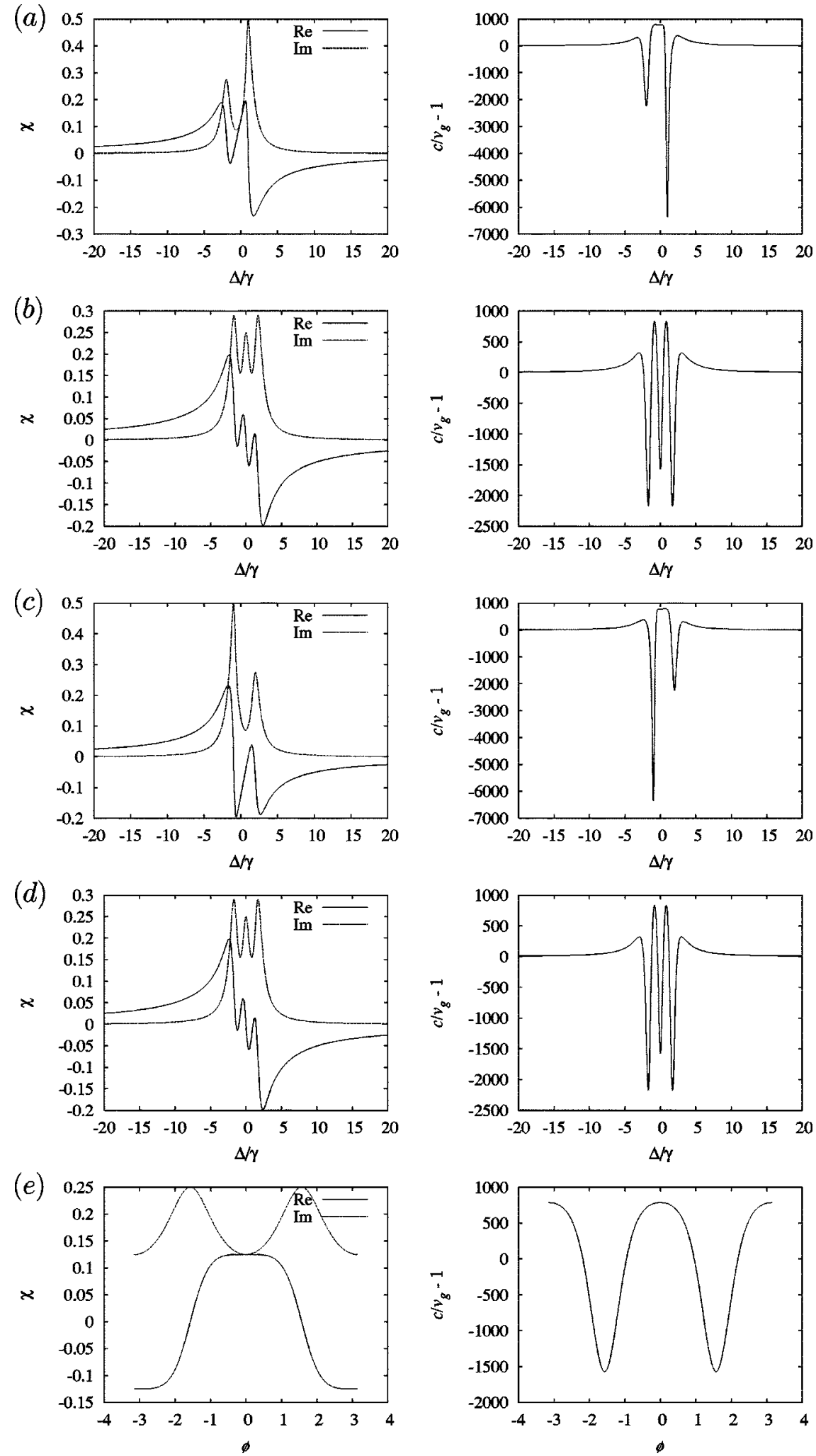

FIG. 2. Phase variation of group velocity (accompanied by absorption). The general parameters are $\Omega_{1}=\Omega_{2}=\Omega_{3}=2 \gamma, \gamma_{1}=\gamma_{2}=2 \gamma$. (a) $\phi=0$, (b) $\phi=\pi / 2$, (c) $\phi=\pi$, (d) $\phi=3 \pi / 2$, and (e) variation of group index as a function of $\phi$.

vacuum and the group velocity $v_{g}$ is given by

$$
v_{g}=\frac{c}{1+2 \pi \chi^{\prime}\left(\nu_{p}\right)+2 \pi \nu_{p} \partial \chi^{\prime}\left(\nu_{p}\right) / \partial \nu_{p}} .
$$

In the following we summarize our results for the dependence of the real and imaginary parts of the susceptibility $\chi$ (i.e., $\chi^{\prime}$ and $\chi^{\prime \prime}$ ) and the group index on the amplitudes and phase of the control fields. 

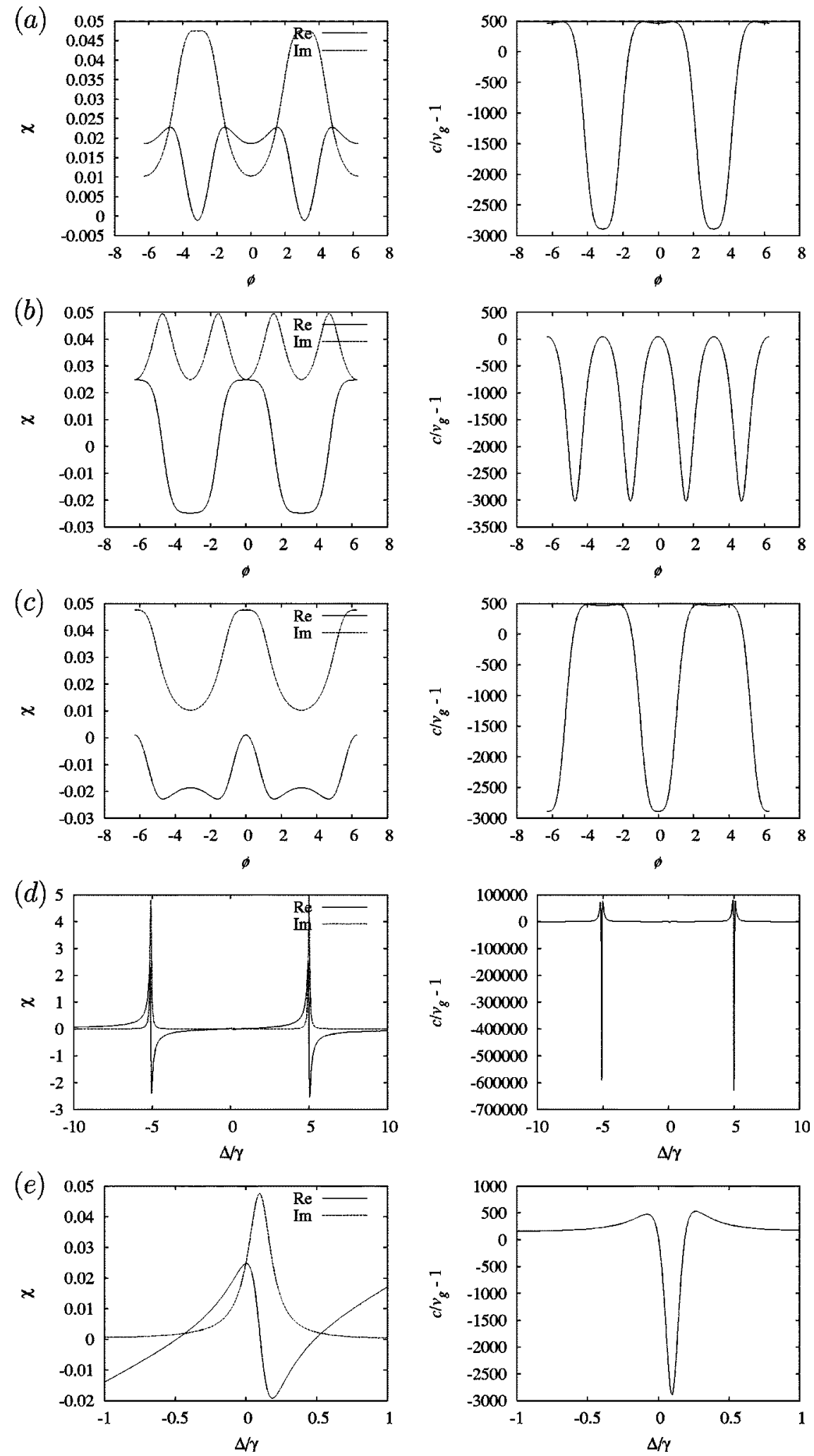

FIG. 3. Phase variation of group velocity (small absorption). The general parameters are $\Omega_{1}=10 \gamma, \Omega_{2}=\Omega_{3}=\gamma, \gamma_{1}=\gamma_{2}=0.2 \gamma$ (a) $\Delta$ $=0.1 \gamma$, (b) $\Delta=0 \gamma$, (c) $\Delta=-0.1 \gamma$, (d) variation as a function of $\Delta / \gamma$ for $\phi=0$. We concentrate on the small feature near $\Delta / \gamma=0$ in the susceptibility curves to obtain (a)-(c). This small feature seen in (d) is magnified and depicted in (e). Thus have superluminality at a considerably less absorption compared to most of the existing proposals. Another point to be noted here is that large variation of group velocity is available for $\Delta / \gamma \neq 0$ but close to 0 . 

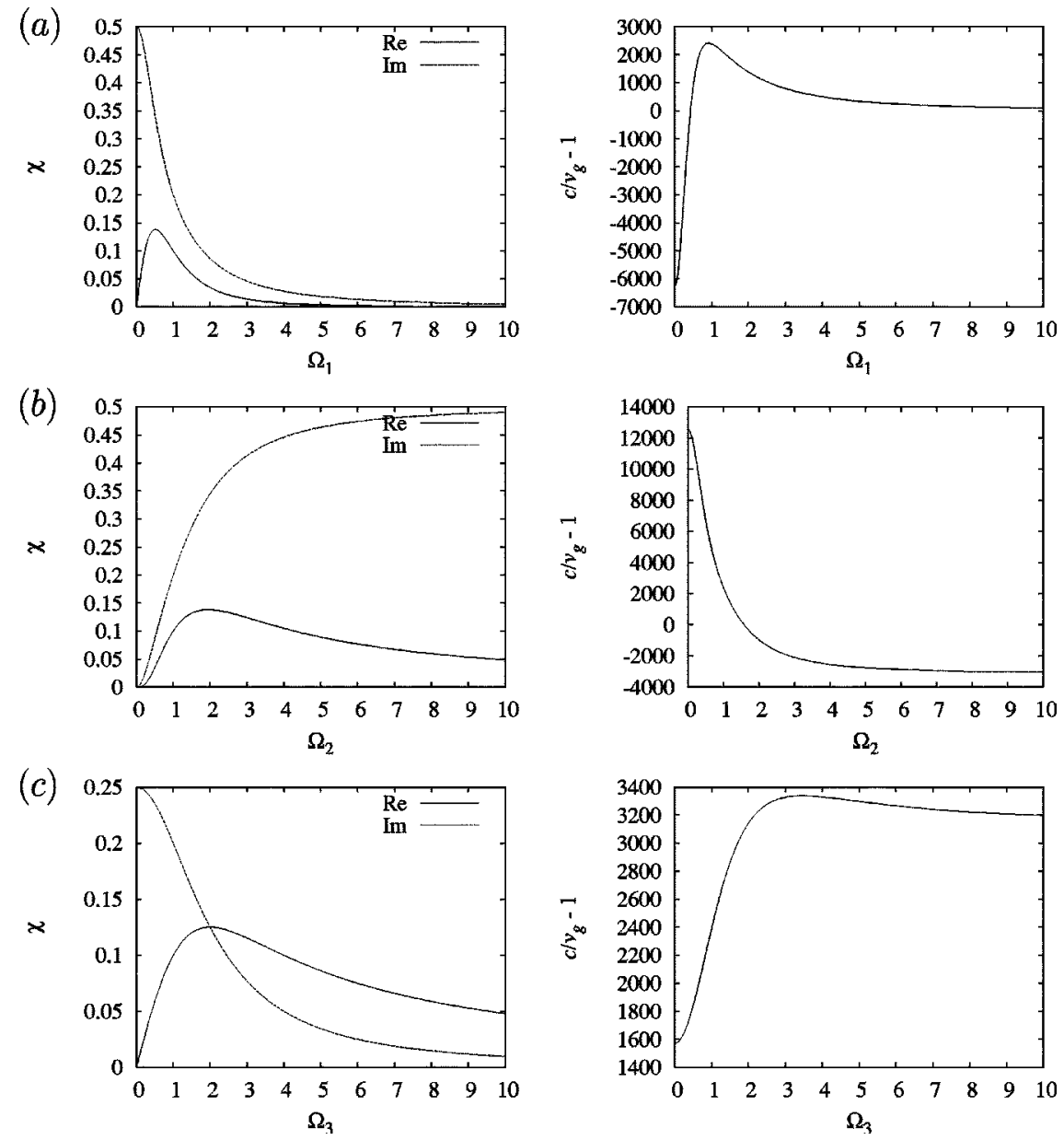

FIG. 4. Intensity tuning of group velocity: The general parameters are $\Omega_{1}=\Omega_{2}=\Omega_{3}=\gamma, \gamma_{1}=\gamma_{2}=2 \gamma$. (a) Variation vs $\Omega_{1}$, (b) variation vs $\Omega_{2}$, (c) variation vs $\Omega_{3}$. We note that only $\Omega_{2}$ provides maximum control over the group velocity of the probe pulse, as it allows switching the group velocity from subliminal to superluminal very much like the observation of Agarwal et al.

We observe that our model imparts unprecedented control over the group velocity of the probe pulse. The group velocity shows continuous tunability over a wide range of values ranging from subluminal to superluminal with just the change of the phase of one of the control fields while other parameters are kept constant. Important feature of our model being considerably less absorption accompanying the superluminal group velocities. Except for the first experimental realization [4] where superluminality was gain assisted, most of the other proposals observe superluminality along with considerable absorption of the pulse as it passes through a specially prepared medium. We discuss our results with the help of Figs. 2-4.

In Fig. 2(a)-2(d) we plot the susceptibility $(\chi)$ and the group index $\left(c / v_{g}-1\right)$ as we vary the phase $(\phi)$ of the field corresponding to the Rabi frequency $\Omega_{3}$. We observe the appearance and disappearance of a peak in the absorption profile in the center near $(\Delta / \gamma=0)$. Thus there is resulting change in the sign of the slope of the dispersion with the phase giving rise to switch in the group velocity of the probe pulse from subluminal to superluminal. This change is continuous as depicted in Fig. 2(e). In this choice of parameters one has superluminality accompanied by slight absorption. Note that we have chosen a modest value of the frequency of the probe field $\nu_{p}=1000 \gamma$. This turn being the dominating one in the group index, for real experimental parameters we expect the range of variation of the group velocity to be much larger than shown here. The susceptibility and $n_{g}-1$ are plotted in the units of $2 N\left|\wp_{a_{1} c}\right|^{2} / \epsilon_{0} \hbar$.

However, it would be desirable to have superluminal propagation with reduced absorption to make sure that the pulse is not attenuated considerably as it passes through the medium. We consider a different range of parameters in Fig. 3 which gives us essentially in the same feature. To illustrate, we observe that the susceptibility has a small feature near the line center $(\Delta / \gamma=0)$ as shown in part (d) of the figure. We concentrate on this small feature and take advantage of the fact that the absorption is small to show phase tuning of the group velocity in parts (a)-(c). It turns out that having the probe field slightly detuned from the probe transition gives a wider range of group velocities [parts (a) and (c)] compared to zero detuning [part (b)]. It is also instructive to notice that even though we are focusing on a small spectral feature of the medium the range of the group velocities available is no less than that observed in Fig. 2.

Another desirable feature in the group velocity control is intensity dependent tunability, proposed by Agarwal et al. [13]. Our model shows the intensity controlled tuning of the 
group velocity as well. The results are summarized in Fig. 4 . Out of the three fields designated by $\Omega_{1}, \Omega_{2}$, and $\Omega_{3}, \Omega_{2}$ provides a wide range of tunability and switching from subluminal to superluminal group velocity. This is understandable as it is the field coupling the forbidden transition as in the case of the proposal by Agarwal et al. [13].

Most of the proposals for the tunability of the group velocity are affected adversely due to the Doppler broadening of the medium. As pointed out in Ref. [17] the tunability from subluminal to superluminal is lost completely due to the Doppler broadening effects in some cases. It is, however, instructive to note that the model we propose here is naturally Doppler free if we consider all the drive fields and the probe field to be propagating colinearly. To put this in perspective we note that the major requirements of our model constitute maintaining the loop formed by the three driving fields and the two-photon resonance condition of EIT among the driving field $\Omega_{1}$ and the probe field. We note that since $\nu_{1}=\nu_{2}+\nu_{3}$, the Doppler shifts for collinear propagation would satisfy $\Delta \nu_{1}=\Delta \nu_{2}+\Delta \nu_{3}$ maintaining the loop structure and phase sensitivity. With the assumption that the low-lying levels $|b\rangle$ and $|c\rangle$ are very close to each other the frequency shift in the driving field corresponding to $\Omega_{1}$ and the probe field are nearly the same thus maintaining the two-photon resonance with the transition $|b\rangle-|c\rangle$. The only requirement for keeping the EIT medium Doppler free is to have sufficiently strong driving field [19]. Thus noting that we work very close to the EIT condition our model is naturally Doppler free and there is very little absorption of any of the fields shining on the medium. Moreover, the model maintains the phase sensitivity as it preserves the loop structure even with the Doppler shifts.

To summarize, we have investigated the effects of the phase and amplitude of the driving fields on the absorption, dispersion and propagation properties of a probe field applied to a specially prepared four-level atom. The model shows a wide range of tunability in the group velocity of the probe field just by changing the phase of one of the control fields. The group velocity can also be switched from subluminal to superluminal through a continuous change of the phase. In contrast with most of the proposals where superluminality is accompanied with considerable absorption, we can reduce the absorption of the probe pulse substantially. The model imparts control of the propagation properties of the probe pulse by controlling the intensities of the laser fields as well. In addition, the model imparts natural Doppler-free situation when all the fields are propagating in a collinear fashion. Moreover, we work within the EIT domain thus with sufficiently strong drive fields one does not have to worry about the absorption of any of the fields incident on the medium.

\section{ACKNOWLEDGMENTS}

The authors wish to thank Y. Rostovtsev for helpful comments and useful discussions. This research was supported in part by the Air Force Office of Scientific Research, the Air Force Research Laboratories (Rome, New York), DARPAQuIST, and the TAMU Telecommunication and Informatics Task Force (TITF) Initiative.

\section{APPENDIX: DETAILS OF THE SUSCEPTIBILITY CALCULATIONS}

We outline the important steps in determination of the density-matrix equation and their solution.

The general form of the density-matrix equations is

$$
\dot{\rho}=-\frac{i}{\hbar}[H, \rho]-\frac{1}{2}\{\Gamma, \rho\}
$$

as already discussed in the manuscript.

Thus the $(i, j)$ the element of the density matrix satisfies the equation

$$
\dot{\rho}_{i j}=-\frac{i}{\hbar} \sum_{k}\left(H_{i k} \rho_{k j}-\rho_{i k} H_{k j}\right)-\frac{1}{2} \sum_{k}\left(\Gamma_{i k} \rho_{k j}+\rho_{i k} \Gamma_{k j}\right) .
$$

Here the indices $i, j$, and $k$ run over $a_{1}, a_{2}, b$, and $c$. The matrix elements of the Hamiltonian can be easily read from the Hamiltonian [see Eqs. (1)-(3)]. The decay matrix has only two nonzero elements given by

$$
\Gamma_{a_{1} c}=\gamma_{1}, \quad \Gamma_{a_{2} c}=\gamma_{2} .
$$

Thus one arrives at the following set of equations for the coherences, i.e., off-diagonal density-matrix elements:

$$
\begin{aligned}
\dot{\rho}_{a_{1} a_{2}}= & -\left(i \omega_{a_{1} a_{2}}+\frac{1}{2}\left(\gamma_{1}+\gamma_{2}\right)\right) \rho_{a_{1} a_{2}}-\frac{i}{2} \Omega_{3} e^{-i \nu_{3} t}\left(\rho_{a_{1} a_{1}}\right. \\
& \left.-\rho_{a_{2} a_{2}}\right)+\frac{i}{2} \Omega_{1} e^{-i \nu_{1} t} \rho_{b a_{2}}-\frac{i}{2} \Omega_{2} e^{i \nu_{2} t} \rho_{a_{1} b} \\
& +\frac{i}{2} \Omega_{p} e^{-i \nu_{p} t} \rho_{c a_{2}}, \\
\dot{\rho}_{a_{1} b}= & -\left(i \omega_{a_{1} b}+\frac{1}{2} \gamma_{1}\right) \rho_{a_{1} b}-\frac{i}{2} \Omega_{1} e^{-i \nu_{1} t}\left(\rho_{a_{1} a_{1}}-\rho_{b b}\right) \\
& -\frac{i}{2} \Omega_{2} e^{-i \nu_{2} t} \rho_{a_{1} a_{2}}+\frac{i}{2} \Omega_{3} e^{-i \nu_{3} t} \rho_{a_{2} b}+\frac{i}{2} \Omega_{p} e^{-i \nu_{p} t} \rho_{c b}, \\
\dot{\rho}_{a_{2} b}= & -\left(i \omega_{a_{2} b}+\frac{1}{2} \gamma_{2}\right) \rho_{a_{2} b}-\frac{i}{2} \Omega_{2} e^{-i \nu_{2} t}\left(\rho_{a_{2} a_{2}}-\rho_{b b}\right) \\
& -\frac{i}{2} \Omega_{1} e^{-i \nu_{1} t} \rho_{a_{2} a_{1}}+\frac{i}{2} \Omega_{3}^{*} e^{i \nu_{3} t} \rho_{a_{1} b}, \\
\dot{\rho}_{a_{1} c}=- & \left(i \omega_{a_{1} c}+\frac{1}{2} \gamma_{1}\right) \rho_{a_{1} c}+\frac{i}{2} \Omega_{3} e^{-i \nu_{3} t} \rho_{a_{2} c}+\frac{i}{2} \Omega_{1} e^{-i \nu_{1} t} \rho_{b c} \\
- & \frac{i}{2} \Omega_{p} e^{-i \nu_{p} t}\left(\rho_{a_{1} a_{1}}-\rho_{c c}\right), \\
- & \frac{i}{2} \Omega_{p} e^{-i \omega_{p} t} \rho_{a_{2} a_{1}}, \\
& \left(i \frac{1}{2} \gamma_{2}\right) \rho_{a_{2} c}+\frac{i}{2} \Omega_{3}^{*} e^{i \nu_{3} t} \rho_{a_{1} c}+\frac{i}{2} \Omega_{2} e^{-i \nu_{2} t} \rho_{b c} \\
\dot{\rho}_{a_{1}} &
\end{aligned}
$$




$$
\begin{aligned}
\dot{\rho}_{b c}= & -i\left(\omega_{b c}+\gamma_{b c}\right) \rho_{b c}+\frac{i}{2} \Omega_{1} e^{i \nu_{1} t} \rho_{a_{1} c}+\frac{i}{2} \Omega_{2} e^{i \nu_{2} t} \rho_{a_{2} c} \\
& -\frac{i}{2} \Omega_{p} e^{-i \nu_{p} t} \rho_{b a_{1}} .
\end{aligned}
$$

Similarly, one can obtain the equations for the level populations, i.e., the diagonal density-matrix elements. Note that $\omega_{i k}$ correspond to the energy difference between the levels $i$ and $k$ and $\nu_{k}$ is the frequency of the $k$ th optical field.

To remove the exponential frequency-dependent factors, one transforms these equations into the rotating frame governed by

$$
\begin{gathered}
\rho_{c a_{2}}=e^{i\left(\nu_{p}-\nu_{3}\right) t} \widetilde{\rho}_{c a_{2}}, \\
\rho_{a_{1} b}=e^{-i\left(\nu_{2}+\nu_{3}\right) t} \widetilde{\rho}_{a_{1} b}, \\
\rho_{a_{2} b}=e^{-i \nu_{2} t} \widetilde{\rho}_{a_{2} b}, \\
\rho_{a_{1} a_{2}}=e^{-i \nu_{3} t} \widetilde{\rho}_{a_{1} a_{2}}, \\
\rho_{b c}=e^{i\left(\nu_{1}-\nu_{p}\right) t} \widetilde{\rho}_{b c}, \\
\rho_{a_{1} c}=e^{-i \nu_{p} t} \widetilde{\rho}_{a_{1} c} .
\end{gathered}
$$

The transformed density-matrix equations take the following form:

$$
\begin{aligned}
\dot{\tilde{\rho}}_{a_{1} a_{2}}= & -\left[i\left(\omega_{a_{1} a_{2}}-\nu_{3}\right)+\frac{1}{2}\left(\gamma_{1}+\gamma_{2}\right)\right] \widetilde{\rho}_{a_{1} a_{2}}-\frac{i}{2} \Omega_{3}\left(\widetilde{\rho}_{a_{1} a_{1}}\right. \\
& \left.-\tilde{\rho}_{a_{2} a_{2}}\right)+\frac{i}{2} \Omega_{1} \widetilde{\rho}_{b a_{2}}-\frac{i}{2} \Omega_{2} \rho_{a_{1} b}+\frac{i}{2} \Omega_{p} \widetilde{\rho}_{c a_{2}}, \\
\dot{\tilde{\rho}}_{a_{1} b}= & -\left[i\left(\omega_{a_{1} b}-\nu_{1}\right)+\frac{1}{2} \gamma_{1}\right] \widetilde{\rho}_{a_{1} b}-\frac{i}{2} \Omega_{1}\left(\widetilde{\rho}_{a_{1} a_{1}}-\widetilde{\rho}_{b b}\right) \\
& -\frac{i}{2} \Omega_{2} \tilde{\rho}_{a_{1} a_{2}}+\frac{i}{2} \Omega_{3} \tilde{\rho}_{a_{2} b}+\frac{i}{2} \Omega_{p} \tilde{\rho}_{c b}, \\
\dot{\rho}_{a_{2} b}= & -\left[i\left(\omega_{a_{2} b}-\nu_{2}\right)+\frac{1}{2} \gamma_{2}\right] \widetilde{\rho}_{a_{2} b}-\frac{i}{2} \Omega_{2}\left(\widetilde{\rho}_{a_{2} a_{2}}-\widetilde{\rho}_{b b}\right) \\
& -\frac{i}{2} \Omega_{1} \widetilde{\rho}_{a_{2} a_{1}}+\frac{i}{2} \Omega_{3}^{*} \widetilde{\rho}_{a_{1} b},
\end{aligned}
$$

$$
\begin{aligned}
\dot{\rho}_{a_{1} c}= & -\left[i\left(\omega_{a_{1} c}-\nu_{p}\right)+\frac{1}{2} \gamma_{1}\right] \widetilde{\rho}_{a_{1} c}+\frac{i}{2} \Omega_{3} \widetilde{\rho}_{a_{2} c}+\frac{i}{2} \Omega_{1} \widetilde{\rho}_{b c} \\
& -\frac{i}{2} \Omega_{p}\left(\widetilde{\rho}_{a_{1} a_{1}}-\widetilde{\rho}_{c c}\right), \\
\dot{\rho}_{a_{2} c}=- & {\left[i\left[\omega_{a_{2} c}-\left(\nu_{p}-\nu_{3}\right)\right]+\frac{1}{2} \gamma_{2}\right] \widetilde{\rho}_{a_{2} c}+\frac{i}{2} \Omega_{3}^{*} \widetilde{\rho}_{a_{1} c}+\frac{i}{2} \Omega_{2} \widetilde{\rho}_{b c} } \\
- & \frac{i}{2} \Omega_{p} \widetilde{\rho}_{a_{2} a_{1}}, \\
\dot{\rho}_{b c}= & -\left[i\left[\omega_{b c}-\left(\nu_{p}-\nu_{1}\right)\right]+\gamma_{b c}\right] \widetilde{\rho}_{b c}+\frac{i}{2} \Omega_{1} \widetilde{\rho}_{a_{1} c}+\frac{i}{2} \Omega_{2} \widetilde{\rho}_{a_{2} c} \\
& -\frac{i}{2} \Omega_{p} \widetilde{\rho}_{b a_{1}} .
\end{aligned}
$$

At this stage we introduce

$$
\Delta=\omega_{a_{1} c}-\nu_{p}=\omega_{a_{2} c}+\nu_{3}-\nu_{p}=\omega_{b c}+\nu_{1}-\nu_{p}
$$

to obtain Eq. (5) which after linearization reduces to Eq. (7).

We now consider the details of solving Eq. (7). It can be written in a form

$$
\dot{R}=-M R+C,
$$

where $R$ and $C$ are column vectors and $M$ is a matrix as given below,

$$
\begin{gathered}
R=\left(\begin{array}{ccc}
\tilde{\rho}_{a_{1} c} & \tilde{\rho}_{a_{2} c} & \tilde{\rho}_{b c}
\end{array}\right)^{\mathrm{T}}, \\
C=\left(\begin{array}{ccc}
\frac{i}{2} \Omega_{p} & 0 & 0
\end{array}\right)^{\mathrm{T}}, \\
M=\left(\begin{array}{ccc}
\left(i \Delta+\gamma_{1} / 2\right) & -\frac{i}{2}\left|\Omega_{3}\right| e^{-i \phi} & -\frac{i}{2} \Omega_{1} \\
-\frac{i}{2}\left|\Omega_{3}\right| e^{i \phi} & \left(i \Delta+\gamma_{2} / 2\right) & -\frac{i}{2} \Omega_{2} \\
-\frac{i}{2} \Omega_{1} & -\frac{i}{2} \Omega_{2} & i \Delta
\end{array}\right) .
\end{gathered}
$$

The formal solution of such a equation is given by

$$
R(t)=\int_{-\infty}^{t} e^{-M\left(t-t^{\prime}\right)} C \mathrm{~d} t^{\prime}=M^{-1} C .
$$

We use the above equation (A10) to obtain the solution for $\tilde{\rho}_{a_{1} c}$ given in Eq. (8).
[1] For excellent reviews, see R. Y. Chiao, and A. M. Steinberg, in Progress in Optics, edited by E. Wolf (Elsevier, Amsterdam, 1997), Vol. 37, p. 345; and R. W. Boyd and D. J. Gauthier, in Progress in Optics, edited by E. Wolf (Elsevier, Amsterdam, 2002), Vol. 43, p. 497.

[2] N. G. Basov, R. V. Ambertsumyan, V. S. Zuev, P. G. Kryukov, and V. S. Letokhov, Sov. Phys. JETP 23, 16 (1966).

[3] A. M. Steinberg and R. Y. Chiao, Phys. Rev. A 49, 2071 (1994).

[4] L. J. Wang, A. Kuzmich, and A. Dogariu, Nature (London) 406, 277 (2000).

[5] M. S. Bigelow, N. N. Lepeshkin, and R. W. Boyd, Science 
301, 200 (2003).

[6] S. E. Harris, J. E. Field, and A. Kasapi, Phys. Rev. A 46, R29 (1992).

[7] L. V. Hau, S. E. Harris, Z. Dutton, and C. H. Behroozi, Nature (London) 397, 594 (1999).

[8] M. M. Kash, V. A. Sautenkov, A. S. Zibrov, L. Hollberg, G. R. Welch, M. D. Lukin, Y. Rostovtsev, E. S. Fry, and M. O. Scully, Phys. Rev. Lett. 82, 5229 (1999).

[9] D. Budker, D. F. Kimball, S. M. Rochester, and V. V. Yashchuk, Phys. Rev. Lett. 83, 1767 (1999).

[10] A. V. Turukhin, V. S. Sudarshanam, M. S. Shahriar, J. A. Musser, B. S. Ham, and P. R. Hemmer, Phys. Rev. Lett. 88, 023602 (2001).

[11] D. Bortman-Arbiv, A. D. Wilson-Gordon, and H. Friedmann, Phys. Rev. A 63, 043818 (2001).

[12] A. D. Wilson-Gordon and H. Friedmann, J. Mod. Opt. 49, 125
(2002).

[13] G. S. Agarwal, T. N. Dey, and S. Menon, Phys. Rev. A 64, 053809 (2001)

[14] A. Wicht, R.- H. Rinkleff, L. S. Molella, and K. Danzmann, Phys. Rev. A 66, 063815 (2002).

[15] F. Ghafoor, S.- Y. Zhu, and M. S. Zubairy, Phys. Rev. A 62, 013811 (2000).

[16] F. Ghafoor, S. Qamar and M. S. Zubairy, Phys. Rev. A 65, 043819 (2002)

[17] C. Goren, A. D. Wilson-Gordon, M. Rosenbluh, and H. Friedmann, Phys. Rev. A 68, 043818 (2003).

[18] M. D. Lukin, S. F. Yelin, M. Fleischhauer, and M. O. Scully, Phys. Rev. A 60, 3225 (1999).

[19] A. B. Matsko, O. Kocharovskaya, Y. Rostovtsev, G. R. Welch, A. S. Zibrov, and M. O. Scully, Adv. At., Mol., Opt. Phys. 46, 191 (2001). 